\title{
Efeitos do Propofol sobre o Sistema de Condução Cardíaca
}

\author{
Paulo Warpechowski ${ }^{1}$, Ari Tadeu Lírio dos Santos, TSA ${ }^{1}$, Paulo José Irigon Pereira ${ }^{2}$, Gustavo Glotz de Lima ${ }^{3}$
}

Resumo: Warpechowski P, Santos ATL, Pereira PJI, Lima GG - Efeitos do Propofol sobre o Sistema de Condução Cardíaco.

Justificativa e objetivos: Alguns estudos têm demonstrado que o uso do propofol é, algumas vezes, associado a bradiarritmias ou reversão de arritmias para ritmo sinusal. Essa propriedade do propofol sugere interferência no Sistema de Condução Cardíaco (SCC).

Conteúdo: Realizou-se revisão com base nos principais artigos contemporâneos que englobam o uso do propofol na presença de arritmias cardíacas. Os autores discorrem sobre os mecanismos fisiopatológicos das taquiarritmias supraventriculares (TSV) e as eventuais interferências causadas pelo propofol sobre o SCC.

Conclusões: Os estudos realizados até então parecem indicar que, provavelmente, o propofol interfere nas TSV automáticas (pelo menos em crianças), mas não nas taquiarritmias em que o mecanismo seja de reentrada.

Unitermos: ANESTÉSICOS, Venoso: propofol; COMPLICAÇÕES: disritmia cardíaca, taquicardia supraventricular.

[Rev Bras Anestesiol 2010;60(4): 438-444] CElsevier Editora Ltda.

\section{INTRODUÇÃO}

Vários fármacos utilizados em anestesia interferem no ritmo cardíaco. Esses efeitos dos anestésicos sobre o Sistema de Condução Cardíaco (SCC), em geral, são bem conhecidos, como aqueles promovidos por fármacos como a succinilcolina ou os opioides. Por outro lado, fármacos mais recentes carecem de estudos minuciosos e seus efeitos não são completamente entendidos. É o caso do propofol, anestésico bastante difundido em nosso meio cujas ações sobre o SCC demandam maior atenção, o que propomos nesta breve revisão. A succinilcolina, bloqueador neuromuscular que guarda semelhança estrutural com a acetilcolina (ACh), mimetiza os efeitos desta sobre os receptores nicotínicos e muscarínicos, levando ao aumento tanto do tônus parassimpático como do simpático. Essa é, provavelmente, a causa de disritmias cardíacas que ocorrem após seu uso. Dentre as principais disritmias estão: a bradicardia sinusal, o ritmo juncional e as disritmias ventriculares, porém são descritas ocorrências desde extrassístoles até fibrilação ventricular ${ }^{1}$. A bradicardia sinusal é o efeito cardiovascular predominante da succinilcolina. Observa-se o ritmo juncional quando a frequência cardíaca se torna menor que a frequência do nó sinusal. Essa disritmia está, em geral, relacionada a um grande estímulo colinérgico

Recebido do Instituto de Cardiologia de Porto Alegre-ICFUC.

1. Anestesiologista do SANE; Mestre em Ciências da Saúde (Cardiologia)

2. ME - Residente do SANE

3. Professor Adjunto do Departamento de Clínica Médica da UFCSPA; Doutor em Ciências da Saúde (Cardiologia); Chefe do Departamento de Eletrofisiologia do ICFUC-Poa

Submetido em 28 de outubro de 2009

Aprovado para publicação em 4 de abril de 2010

Endereço para correspondência:

Dr. Paulo Warpechowski

Rua Vitor Meireles 200, 804

Rio Branco

90430-160 - Porto Alegre, RS

E-mail: paulowarpe@gmail.com no nível do nó sinusal, o qual causa supressão da atividade sinusal e propicia o surgimento de marca-passo no nó atrioventricular (AV) ${ }^{1,2}$.

Por outro lado, alguns efeitos cardíacos dos anestésicos podem ser benéficos, como o efeito vagotônico central e a bradicardia que ocorre após a administração de opioides, tendo como resultante menor consumo metabólico cardíaco ${ }^{3}$. Os opioides também afetam os canais de cálcio e potássio cardíaco, prolongando o potencial de ação. Esse quadro suporta evidência de alguma atividade antiarrítmica desses fármacos, semelhantes aos agentes antiarrítmicos da classe III 4,5.

O propofol, agente hipnótico largamente usado como sedativo, hipnótico e coadjuvante de anestesia venosa, eventualmente promove bradiarritmias e conversão de taquiarritmias para o ritmo sinusal ${ }^{5,6}$, o que sugere que esse fármaco interfere no SCC. Os mecanismos citados para essa ocorrência são os mais diversos e incluem efeitos eletrofisiológicos diretos sobre o SCC ou efeitos indiretos, como alterações no tônus do sistema nervoso autônomo (SNA) e alterações ácidobásicas ${ }^{9,10}$. $\mathrm{O}$ esclarecimento do mecanismo gerador desses eventos é de elevada importância, pois determina a indicação ou não desse fármaco em determinadas situações clínicas.

Dentre as disritmias envolvidas nesses relatos clínicos, as mais comuns são as taquicardias supraventriculares (TSV), que serão descritas a seguir.

\section{Mecanismos fisiopatológicos das taquicardias supraventriculares}

Os dois mecanismos básicos responsáveis pela geração das TSV são o aumento da automaticidade e as anormalidades de condução, levando à reentrada 7 .

A alteração da automaticidade, ou aumento da geração do impulso, pode resultar do aumento da automaticidade da fase 4 em células normais ou anormais (automaticidade 
anormal). Pode ocorrer também por conta de repetidos disparos de pós-potenciais, presentes nas fases 3 ou 4 do potencial de ação (atividade deflagrada aumentada). Entre os diversos fatores causadores da automaticidade aumentada, as alterações metabólicas são as mais frequentes. Podem-se citar ainda aumento de catecolaminas circulantes, hipoxemia, hipercarbia, hipocalemia aguda, hipomagnesemia, alterações na tensão da parede miocárdica e isquemia miocárdica ${ }^{3,7}$.

O mecanismo reentrante pode ser classificado como anatômico, funcional ou ainda como a combinação de anatômico e funcional (anisotrópico). Exemplos do mecanismo reentrante incluem fibrilação atrial, taquicardia por reentrada nodal atrioventricular (TRN) e TSV por feixe anômalo (acessório). Esta última tem como exemplo a síndrome de Wolff Parkinson-White (WPW) ${ }^{7,8}$.

A TRN é causada por mecanismo reentrante que ocorre nas proximidades do nó atrioventricular (AV). Para que o mecanismo seja desencadeado, são necessárias duas ou mais vias com diferentes tempos de condução e períodos refratários distintos. Uma delas é chamada de via lenta (ou alfa) e tem condução lenta e período refratário curto. A outra, de condução rápida e período refratário longo, é denominada via rápida (ou beta). É necessário também que ocorra bloqueio unidirecional em uma dessas vias, em geral na via rápida. Quando um estímulo (na maioria das vezes, uma extrassístole atrial) desce pela via lenta e alcança a via rápida em um momento que seja possível a condução retrógrada gera-se batimento de eco nodal. Assim, o atraso da condução e um período refratário apropriado nas duas vias geram um circuito reentrante. Se isso ocorrer de maneira continuada, o fenômeno dará origem à TRN do tipo comum, característico de $90 \%$ dos casos de TRN. Já na TRN do tipo incomum, ocorre o inverso, ou seja, o estímulo desce pela via rápida e segue retrogradamente pela via lenta ${ }^{7,8}$.

A TRN é o tipo de TSV mais comum, ocorrendo em aproximadamente $50 \%$ dos casos. É mais frequente em mulheres e, geralmente, ocorre antes dos 40 anos ${ }^{7,8}$. Nessa disritmia, a frequência cardíaca pode variar de 100 a 280 bpm, sendo a média de $170 \mathrm{bpm}$. Tanto a TRN quanto a TSV por feixes anômalos são passiveis de cura por meio de ablação por radiofrequência (RF) através de cateter percutâneo 7,8 .

\section{Farmacologia}

O propofol é um agente anestésico venoso amplamente utilizado em anestesia geral e como sedativo em procedimentos para exames diagnósticos ou terapêuticos, como, por exemplo, durante estudo eletrofisiológico (EEF) e para ablação por RF, devido às suas propriedades farmacocinéticas favoráveis, como por exemplo, despertar rápido, ausência de efeitos cumulativos e facilidade de titulação 9,10,14. Porém, pode promover redução da pressão arterial (PA) e da resistência vascular periférica (RVP) e essas alterações, em geral, não são seguidas por aumento compensatório da frequência cardíaca (FC) 9,10,16.

Essa falta de compensação da FC, o relato de bradiarritmias ${ }^{11}$, a supressão de taquiarritmias ${ }^{5,6}$ e a conversão de outros ritmos para o sinusal durante o uso do propofol apontaram para a possibilidade de ocorrência de bloqueio dos barorreceptores ou de depressão do SCC causados por esse fármaco ${ }^{6,15}$. Vários relatos de ocorrência de bradicardia, bloqueio do SCC e reversão de taquiarritmias para ritmo sinusal, após o uso do propofol, têm sido publicados na literatura 5,6,11-13.

Alguns autores sugeriram que o propofol promove supressão de taquicardia atrial (supraventricular) e referem que esse fármaco deveria ser evitado durante os procedimentos de EEF ${ }^{14}$.

Uma vasta revisão sistemática sobre o propofol entre os anos de 1984 e 1995 encontrou 65 artigos e 187 relatos com diferentes graus de evidência para a indução de bradicardia, totalizando 1.444 bradicardias, 86 assistolias e 24 mortes relacionadas ao seu uso. Dentre os estudos controlados revisados por esses autores, o propofol aumentou significativamente o risco de bradicardia em comparação com outros anestésicos, resultando em um cálculo do "number-neededto-harm" (NNH) de 11,3 (intervalo de confiança de 95\%, de 7,7-21). Durante cirurgias de estrabismo em crianças, o NNH foi de 4,1 (3-6,7). Esses autores concluíram que o risco de morte relacionado com a bradicardia com propofol foi estimado em 1,4:100.000 e o risco de assistolia foi estimado em 15:10.000 ${ }^{15}$.

Esses fatos têm gerado controvérsia a respeito de possíveis efeitos diretos causados pelo propofol sobre o SCC ou se essas alterações de ritmos devem-se a ações indiretas do fármaco.

Vários estudos têm demonstrado que o propofol apresenta ambos os efeitos cardiovasculares, diretos e indiretos ${ }^{9,13,15-18}$.

Os efeitos indiretos incluem modulação do tônus do SNA e alteração da sensitividade do reflexo barorreceptor ${ }^{9}$. Como não é possível demonstrar alguma atividade vagolítica central do propofol e também porque parece que ele exerce efeitos vagotônicos ou simpatolíticos é provável que seja responsável pela ocorrência de bradicardia em alguns pacientes ${ }^{17}$. Esses efeitos indiretos sobre o sistema cardiovascular foram descritos por Deutschman e col., que constataram redução do tônus simpático mais intenso do que aquela que ocorreu com o tônus parassimpático promovido pelo propofol e isso poderia explicar a bradicardia que ocorre em alguns pacientes durante seu uso ${ }^{18}$. Do mesmo modo, Hidaka e col., ao comparar os efeitos do propofol e do midazolam sobre o SNA, encontraram que o propofol exerce efeito simpaticolítico mais potente do que o midazolam sobre o SNA ${ }^{19}$.

Esses estudos corroboram a ideia de um importante efeito indireto sobre o SNA, o que poderia explicar a ocorrência de bradicardia ou a supressão de taquicardia quando se utiliza esse fármaco.

Os efeitos diretos do propofol, que são basicamente aqueles exercidos sobre o SCC ou sobre o músculo cardíaco, têm sido alvo de diversos estudos, como será detaIhado a seguir.

\section{Bases biológicas}

Os efeitos do propofol no SCC têm sido demonstrados em vários estudos realizados em animais. Alphin e col. relataram 
que o propofol atrasa o tempo de condução do nó AV de modo concentração-dependente em cobaias. Além disso, esses autores verificaram que o propofol reduz a frequência atrial e que esses efeitos dromotrópicos negativos são predominantemente mediados pelos receptores muscarínicos M2. Concluíram ainda que, de forma semelhante aos efeitos causados pelos agentes antiarrítmicos diltiazem e adenosina, o propofol parece gerar efeitos diretos e indiretos nas propriedades de condução cardíaca ${ }^{16}$.

Estudo efetuado em porcos demonstrou que a função do sistema His-Purkinje e do nó sinusal foi deprimida de modo dose-dependente pelo propofol. Porém, esses autores não conseguiram demonstrar efeitos do fármaco sobre a função do nó AV ou sobre o tecido de condução atrial ou ventricular ${ }^{20}$.

Já nos estudos realizados em cães, não se observou qualquer efeito direto sobre o SCC quando se realizou bloqueio completo do SNA, com atropina e propranolol, antes da administração do propofol ${ }^{21}$.

Por outro lado, Napolitano e col., estudando as propriedades antiarrítmicas dos anestésicos tionembutal, cetamina e propofol em cobaias, chegaram às seguintes conclusões: 1) o tionembutal prolonga o período refratário efetivo do nó $\mathrm{AV}$, enquanto o propofol e a cetamina não; 2) a cetamina reduziu a velocidade de condução atrial (VCA), mas o propofol e o tionembutal não tiveram efeito sobre a VCA; 3) todos os três anestésicos estudados causaram aumento concentração-dependente do intervalo de condução do feixe de His. Os autores concluíram que o propofol poderia ser mais efetivo para filtrar impulsos atriais durante as TSVs, enquanto o tiopental seria mais efetivo em prevenir disritmias atriais reentrantes ${ }^{22}$.

Wu e col. avaliaram os efeitos desse fármaco em coelhos e comprovaram que o propofol, em doses baixas, promoveu aumento significativo do intervalo de condução AV. Os autores concluíram que, em doses clínicas, o propofol pode modificar diretamente a condução AV. Sugeriram que esse fármaco poderia interferir na indução de taquicardia durante ablação por RF e, portanto, influenciar na decisão terapêutica durante esse procedimento ${ }^{23}$.

\section{Estudos em seres humanos}

Dois estudos que avaliaram o propofol durante anestesias para EEF em humanos não conseguiram demonstrar qualquer efeito direto causado por esse fármaco na atividade do nó sinoatrial, intra-atrial ou na condução AV 24,25.

Da mesma forma, Romano e col. ${ }^{26}$ não conseguiram comprovar efeito do propofol sobre o SCC e também não verificaram a ocorrência de bradiarritmias durante seu uso; pelo contrário, o ciclo sinusal mostrou redução estatisticamente significativa durante o uso do propofol, comparado com o grupo-controle.

Porém, Erb e col., ao comparar o propofol com isoflurano em crianças submetidas a EEF e ablação por RF, verificaram que houve prolongamento da condução nodal AV de maneira estatisticamente significativa. Contudo, os autores concluíram que esse achado não apresentou importância clínica. Segundo eles, os dois fármacos seriam elegíveis para esses procedimentos 27 .
Por outro lado, Wu e col ${ }^{28}$, após utilizarem propofol em anestesia para EEF, relataram que, de nove pacientes pediátricos com taquicardia atrial ectópica, em quatro (44\%) não foi possível sustentar a taquicardia e, portanto, situar sua origem, impedindo o tratamento por ablação durante anestesia com propofol. Com base nisso e em estudos realizados em coelhos ${ }^{23}$, nos quais o propofol prolongou o período refratário atrial e a condução AV, os autores sugeriram evitar o uso da substância em anestesia durante ablação para pacientes com taquicardia atrial ectópica ${ }^{28}$.

Conclusões semelhantes foram publicadas por Lai e col., em uma série de 150 pacientes, em que a maioria (148/152) das taquicardias permaneceu indutível após a anestesia com propofol. No entanto, em quatro de sete pacientes (57\%) pediátricos com taquicardia atrial ectópica, esta terminou após a administração do propofol e não pôde ser induzida, mesmo após a infusão de isoproterenol - fármaco utilizado para facilitar a indução programada dessas disritmias durante o EEF. Os autores sugerem que esse agente anestésico deve ser utilizado com cautela em pacientes pediátricos com taquicardia atrial ectópica submetidos à EEF 29

Em um estudo aleatório, Warpechowski e col. ${ }^{30}$ avaliaram os efeitos do propofol sobre o sistema de condução AV de pacientes com TRN, por meio da análise dos períodos refratários efetivos da via lenta e da via rápida do nó AV durante o EEF. Os autores concluíram que o propofol não promoveu alteração significativa das variáveis eletrofisiológicas do nó AV, resultados que foram semelhantes aos de Sharpe e col. ${ }^{24} \mathrm{e}$ Lavoie e col. ${ }^{25}$. Tais achados não revelam evidência de que o propofol possa agir de maneira direta sobre as propriedades eletrofisiológicas do nó AV em pacientes com TRN. Da mesma forma, o propofol não impediu a indução de taquiarritmias programadas durante o EEF, não interferindo, portanto, no diagnóstico dessas taquiarritmias ${ }^{30}$.

O estudo de Sharpe e col. ${ }^{24}$ incluiu pacientes com síndrome WPW, enquanto o de Warpechowski e col. ${ }^{30}$ avaliou apenas pacientes com TRN, ou seja, ambos estudaram pacientes com disritmia cujo mecanismo era a reentrada. Lavoie e col. ${ }^{25}$ por sua vez, estudaram vinte crianças, dentre as quais 17 tinham vias acessórias (WPW e feixes ocultos), uma apresentava taquicardia juncional reciprocante e duas o diagnóstico de TRN. Com base nesses estudos, é possível concluir que, provavelmente, o propofol não interfere no sistema de condução AV em pacientes em que o substrato da arritmia seja a reentrada.

Entretanto, Wu e col. ${ }^{23}$, com base em estudo realizado em animais nos quais o propofol promoveu efeitos no SCC e também em suas observações sobre os pacientes pediátricos com taquicardia atrial ectópica ${ }^{28}$, em que não foi possível sustentar essa taquicardia em quatro pacientes na vigência do uso de propofol, sugeriram eventual interferência desse fármaco sobre o SCC no grupo de pacientes com esse substrato arritmogênico. Essa conclusão é semelhante à de Lai e col. ${ }^{29}$ que não conseguiram induzir a taquiarritmia mesmo quando facilitada com a infusão de isoproterenol e sugeriram que o propofol, de algum modo, interferiu no mecanismo dessa disritmia, impedindo sua indução programada. 


\section{CONCLUSÃO}

Os estudos realizados até aqui parecem indicar que, provavelmente, o propofol interfere de algum modo nas TSVs automáticas (pelo menos em crianças), mas não nas taquiarritmias em que o mecanismo é de reentrada, como na TRN ou taquicardia dependente de feixe acessório.

Maiores estudos deverão ser realizados para verificar a possibilidade de interferência desse fármaco nas TSVs automáticas. Nesse ínterim, deve-se dedicar especial atenção quando da utilização do propofol nos procedimentos de EEF em pacientes que apresentam essa arritmia, pois poderiam interferir no diagnóstico e, consequentemente, também impedir o tratamento com ablação por RF.

Igualmente, sugere-se a utilização com parcimônia desse fármaco no grupo de pacientes pediátricos submetidos a procedimentos potencialmente arritmogênicos, em especial quando é possível ocorrer bradiarritmia, como, por exemplo, nas cirurgias de correção de estrabismo.

\section{REFERÊNCIAS / REFERENCES}

01. Oliveira LF. Succinilcolina. em: Cavalcanti IL, Diego LAS. Bloqueadores Neuromusculares: Bases Científicas e Uso Clínico em Anestesiologia. São Paulo: EPM, 2002;87-97.

02. Stoelting RK. Neuromuscular-Blocking Drugs. em: Stoelting RK. Pharmacology and Physiology in Anesthetic Practice. $3^{\text {rd }}$ Ed, Philadelphia: Lippincott-Raven, 1999;182-223.

03. Stoelting RK. Opioid Agonists and Antagonists. em: Stoelting RK. Pharmacology and Physiology in Anesthetic Practice. $3^{\text {rd }}$ Ed, Philadelphia: Lippincott-Raven, 1999;77-112.

04. Pruett JK, Blair JR, Adams RJ. Cellular and Subcellular Actions of Opioids in the Heart. em: Estafanous FG. Opioids in Anesthesia. Boston: Butterworth-Heinemann, 1991;61-71.

05. Miró O, de la Red G, Fontanals J. Cessation of paroxysmal atrial fibrillation during acute intravenous propofol administration. Anesthesiology, 2000;92:910.

06. Kannan S, Sherwood N. Termination of supraventricular tachycardia by propofol. Br J Anaesth, 2002;88:874-875.

07. Renwick J, Kerr C, McTaggart R et al. Cardiac electrophysiology and conduction pathway ablation. Can J Anaesth, 1993;40:10531064.

08. Deshpande S, Jazayeri M, Dhala A. Selective Transcatheter Modification of the Atrioventricular Node. em: Zipes DP - Catheter Ablation of Arrhythmias. New York: Futura Publishing, 1994;151-186.

09. Hug CC, McLeskey CH, Nahrwold NL et al. Hemodynamic effects of propofol- data from 24,771 patients. Anesth Analg, 1993;76:S154.

10. Vuyk J, Engbers FHM, Lemmens HJM et al. Pharmacodynamics of propofol in female patients. Anesthesiology, 1992;77:3-9.

11. Thomsom SJ, Yate PM. Bradycardia after propofol infusion. Anaesthesia, 1987;42:430

12. Seki $\mathrm{S}$, Ichimiya $\mathrm{T}$, Tsuchida $\mathrm{H}$ et al. A case normalization of WolffParkinson-White syndrome conduction during propofol anesthesia. Anesthesiology, 1999;90:1779-1781.

13. Burjorjee JE, Milne B. Propofol for electrical storm: a case report of cardioversion and suppression of ventricular tachycardia by propofol. Can J Anaesth, 2002;49:973-977.

14. Reves JG, Glass PSA, Lubarsky DA et al. Intravenous Nonopioid Anesthetics, em: Miller RD - Miller's Anesthesia. $6^{\text {th }}$ Ed, Philadelphia: Elsevier Churchill Livingstone, 2005;318-368.

15. Tramèr MR, Moore RA, Mcquay HJ. Propofol and bradycardia: causation, frequency and severity. Br J Anaesth, 1997;78:642-651.

16. Alphin RS, Martens JR, Dennis DM. Frequency-dependent effects of propofol on atrioventricular nodal conduction in guinea pig isotated heart. Mechanism and potencial antidysrrhythmic properties. Anesthesiology, 1995;83:382-94.

17. Hermann R, Vettermann J. Change of ectopic supraventricular tachycardia to sinus rhythm during administration of propofol. Anesth Analg, 1992;75:1030-1032.

18. Deutschman CS, Harris AP, Fleisher LA. Changes in heart rate variability under propofol anesthesia: a possible explanation for propofolinduced bradycardia. Anesth Analg, 1994;79:373-377.

19. Hidaka $S$, Kawamoto $M$, Kurita $S$ et al. Comparison of the effects of propofol and midazolam on the cardiovascular autonomic nervous system during combined spinal and epidural anesthesia. J Clin Anesth, 2005;17:36-43.

20. Pires LA, Huang SKS, Wagshal AB et al. Electrophysiological effects of propofol on the normal cardiac conduction system. Cardiology, 1996;87:319-324.

21. Ikeno S, Akazawa S, Shimizu R et al. Propofol does not affect the canine cardiac conduction system under autonomic blockade. Can J Anaesth, 1999;46:148-153.

22. Napolitano CA, Raatikainen MJP, Martens JR et al. Effects on intravenous anesthetics on atrial wavelength and atrioventricular nodal conduction in guinea pig heart. Potential antidysrhythmic properties and clinical implications. Anesthesiology, 1996; 85:393-402.

23. Wu MH, Su MJ, Sun SSM. Age-related propofol effects on electrophysiological properties of isolated hearts. Anesth Analg, 1997;84:964-971.

24. Sharpe MD, Dobkowski WB, Murkin JM et al. Propofol has no direct effect on sinoatrial node function or on normal atrioventricular and accessory pathway conduction in Wolf-Parkinson-White syndrome during alfentanil/midazolam anesthesia. Anesthesiology, 1995;82:888-895.

25. Lavoie J, Walsh EP, Burrows FA et al. Effects of propofol or isofluorane anesthesia on cardiac conduction in children undergoing radiofrequency catheter ablation for tachydysrhythmias. Anesthesiology, 1995; 82:884-887.

26. Romano R, Ciccaglioni A, Fattorini $F$ et al. Effects of propofol on the human heart electrical system: a transesophageal pacing electrophysiologic study. Acta Anaesthesiol Scand, 1994;38:30-32.

27. Erb TO, Kanter RJ, Hall JM et al. Comparison of electrophysiologic effects of propofol and isoflurane-based anesthetics in children undergoing radiofrequency catheter ablation for supraventricular tachycardia. Anesthesiology, 2002;96:1386-1394.

28. Wu MH, Lin JL, Lai LP et al. Radiofrequency catheter ablation of tachycardia in children with and without congenital heart disease: indications and limitations. Int J Cardiol, 2000; 72:221-227.

29. Lai LP, Lin JL, Wu MJ. Usefulness of intravenous propofol anesthesia for radiofrequency catheter ablation in patients with tachyarrhythmias: infeasibility for pediatric patients with ectopic atrial tachycardia. Pacing Clin Electrophysiol, 1999; 22:1358-1364.

30. Warpechowski P, Lima GG, Medeiros CM et al. Randomized study of propofol effect on electrophysiological properties of the atrioventricular node in patients with nodal reentrant tachycardia. Pacing Clin Electrophysiol, 2006;29:1375-1382.

Resumen: Warpechowski P, Santos ATL, Pereira PJI, Lima GG Efectos del Propofol sobre el Sistema de Conducción Cardíaco.

Justificativa y objetivos: Algunos estudios han demostrado que el uso del propofol es algunas veces, asociado a bradiarritmias o a reversión de arritmias para ritmo sinusal. Esa propiedad del propofol nos sugiere una interferencia en el Sistema de Conducción Cardíaco (SCC).

Contenido: Se realizó una revisión en base a los principales artículos contemporáneos que engloban el uso del propofol cuando ocurren arritmias cardíacas. Los autores nos hablan sobre los mecanismos fisiopatológicos de las taquiarritmias supraventriculares y las eventuales interferencias causadas por el propofol sobre el SCC.

Conclusión: Los estudios realizados hasta ahora parecen indicar que probablemente, el propofol interfiere en las TSV automáticas (por lo menos en niños), pero no en las taquiarritmias en que el mecanismo sea de reentrada. 